Anales de Geografía de la Universidad Complutense ISSN: 0211-9803

http://dx.doi.org/10.5209/rev_AGUC.2016.v36.n1.52712

\title{
Ejemplos de custodia del territorio en Brasil. El Projecto Sempre Viva (Mucugê-Bahia)
}

\author{
Manuel Cabalar Fuentes ${ }^{1}$; Ricardo Augusto Souza Machado \\ Recibido: 11 de febrero del 2015 / Enviado a evaluar: 15 de febrero del 2015 / Aceptado: 13 de julio del 2015
}

Resumen. En los últimos años ha ganado importancia el concepto de "Custodia del Territorio". En síntesis, implica que tanto entidades locales como particulares pueden proteger enclaves de elevado valor ecológico-cultural. Esta visión ha cuajado en algunos lugares del mundo con señalado éxito. Es el caso del Projeto Sempre Viva, nacido en la localidad de Mucugê, situada en uno de los ENP más renombrados de Brasil: la Chapada Diamantina (Estado de Bahia). Se ha realizado una consulta de las informaciones disponibles sobre el proyecto en Internet, así como una visita personal al lugar para conocerlo y poder hablar con sus gestores. En base a ello, se ha concluido que este proyecto, nacido para proteger una especie endémica de Siempre-viva en peligro de extinción, ha derivado en un importante foco de turismo y educación ambiental, completada con la preservación de la identidad cultural de la región, ligada a la minería del diamante.

Palabras clave: Custodia del Territorio; protección de la naturaleza; gestión local de la conservación; Projeto Sempre Viva; Mucugê; Brasil.

\section{[en] Examples of "land stewarship" in Brazil. The "Projecto Sempre Viva" (Mucugê-Bahia)}

Abstract. In recent years the concept of "Land Stewardship" has gained a great importance. In short, it means that local entities and individuals can protect enclave of high ecological and cultural value. This vision has been effective in some places in the world with success. For example, the "Projeto Sempre Viva", created in the town of Mucugê, a place located in one of Brazil's most renowned Natural Protected Areas: the Chapada Diamantina (State of Bahia). We had looked over the available information about this project on the Internet, as well as a personal visit that was made to the site to know it and to have the possibility to talk to their managers. On this basis, it has been reached to the conclusion that this project, born to protect a Sempre Viva's endemic species in danger of extinction, has turned into an important site for tourism and environmental education, completed with the preservation of the cultural identity of this region which is linked to diamond mining.

Key words: Land Stewardship; protection of nature; local management of conservation; Projeto Sempre Viva; Mucugê; Brazil.

1 Departamento de Ciências Humanas e Filosofia. Universidade Estadual de Feira de Santan (Bahia-Brasil)

E-mail: manuel.cabalar@gmail.com 
[fr] Examples de "garde du territoire" a Brésil. Le Projecto Sempre Viva (Mucugê-Bahia)

Résumé. Dans les dernières années, le concept de "Custodia del Territorio" ("Garde du Territoire”) a gagné beaucoup de force. D’une manière synthétique, ce concept implique que les entités locales et individus puissent protéger les enclaves de grande valeur écologique et culturel. Cette vision a été matérialisée en certains lieux du monde avec une réussite remarquable. C’est le cas du Projeto Sempre Viva ("Projet Toujours Vivant”) né dans la localité de Mucugé et localisé dans un des ENP plus renommés du Brésil: la Chapada Diamantina (État de Bahía). Un recueil des informations disponibles sur Internet et une sortie à ce terrain ont été réalisés pour prendre connaissance des lieux et pour pouvoir parler aux gérants. À partir de cela, nous concluons que ce projet, né pour protéger une espèce endémique de "Siempre-Viva" (“Toujours-Vivante") en danger d'extinction, a été l'origine d'un noyau de tourisme et d'éducation environnementale. Cela a été complété par la préservation de l'identité culturale de la région, liée à l'activité minière du diamant.

Mots clés: Garde du territoire; protection de la nature; gestion locale de la préservation; Projeto Sempre Viva; Mucugê; Brésil.

Cómo citar. Cabalar Fuentes, M. y Souza Machado, R.A. (2016): Ejemplos de custodia del territorio en Brasil. El Projecto Sempre Viva (Mucugê-Bahia). Anales de Geografía de la Universidad Complutense, 36(1), 31-47.

Sumario. 1. Introducción: La conservación de la naturaleza como misión del poder público. 2. Discutiendo el concepto de custodia del territorio. 3. Marco histórico: del Garimpo a la recolección de Siempre-Vivas. 4. Nacimiento del Projecto Sempre Viva. Motivos, actores implicados, objetivos. 5. El Projecto Sempre Viva hoy. 6. Conclusiones. 7. Referencias bibliográficas.

\section{Introducción: La conservación de la naturaleza como misión del poder público}

Habitualmente se cree que la preocupación por preservar la naturaleza por un lado, y el modelo de sociedad urbano-industrial capitalista surgido en Europa a inicios del Siglo XIX y extendido por el Mundo, por otro lado, nacieron al mismo tiempo. Eso sólo es cierto en el sentido de que los cada vez más evidentes impactos sobre la naturaleza y la aparición primero y difusión posterior de Espacios Naturales Protegidos (en adelante, ENP), declarados por actos legales del poder público, son procesos que van unidos en el tiempo, a modo de acción-reacción. Los Parques Nacionales pioneros que se declaran en Estados Unidos tiene como una de sus razones fundacionales la salvaguarda de las últimas representaciones de naturaleza virgen de la mano destructora del Hombre. Las leyes que promulgan su creación así lo recogen, con palabras más o menos similares.

Sin embargo, esto no es fruto de una súbita aparición de una conciencia ambiental. En realidad, en el ser humano ha anidado siempre una percepción innata de la importancia que la naturaleza y sus procesos tienen para su supervivencia. Es así como, desde hace miles de años, diversos lugares, enclaves y territorios han sido preservados y respetados por su importancia como abastecedores de alimento o de agua (lo que actualmente se conoce por "Servicios Ambientales"), amén del valor simbólico atribuido a varios de ellos, hecho directamente relacionado con prácticas religiosas de veneración de las fuerzas naturales, muy comunes en sociedades 
antiguas y aún hoy existentes en grupos humanos más al margen de la influencia de la sociedad occidental dominante (Mulero, 2002; Diego et alt, 2007). Sin embargo, no será hasta el último tercio del Siglo XIX cuando la idea conservacionista acabe siendo asimilada políticamente, en forma de protección de territorios seleccionados ante todo por su grandiosa belleza escénica. No es casualidad pues que los primeros ENP nazcan en los EEUU (Parque Nacional de Yellowstone, 1872), nación embarcada de lleno en la Revolución Industrial y que si por un lado veía ya en sus áreas urbanas las consecuencias de la degradación del medio natural y de la calidad de vida de amplias capas de la población, por otro lado mantenía en su Far West inmensos territorios casi despoblados de gran belleza que se creía necesario dejar al margen de toda intervención humana, como santuarios de la Madre Naturaleza a contemplar en todo su esplendor. Este modelo, con escasas modificaciones, fue trasplantado al resto del mundo en los siguientes decenios. En consecuencia, la existencia de ENP no fue más que la respuesta del poder público al afán humano de poner a salvo la belleza natural, sentimiento que está fuertemente arraigado en la psique humana (Corraliza et alt, 2002), y que aún existe en el fondo de gran parte de las declaraciones de ENP hasta nuestros días. Basta leer los principios y objetivos recogidos en las leyes encargadas de proteger la naturaleza y no será difícil hallar alusiones a la belleza como un motivo para declarar éste o aquel espacio ${ }^{2}$.

Desde entonces son los gobiernos (los Estados-Nación, si se prefiere), incluso las administraciones de escala regional, los encargados de establecer y aprobar todo un marco legal de protección, acompañado de la delimitación territorial de ENP y el establecimiento de sus normas de manejo. Incluso las Constituciones de muchos Estados recogen en su articulado la obligación de conservar la naturaleza en buen estado para el bien común de la sociedad como uno de sus principios rectores. Pero no todo el trabajo puede ni debe ser hecho por la administración pública. La misma sociedad en su conjunto, así como todos y cada uno de sus miembros en la medida de sus posibilidades, deben tomar parte en la misión de mantener un medio ambiente en buenas condiciones. ${ }^{3}$ Esto también viene acompañado de un cambio notable de paradigma en el enfoque de la conservación, que ha conseguido evolucionar de la

2 Por ejemplo, la Ley brasileña que regula su sistema de Unidades de Conservación (Lei 9985, de 18 de Julho de 2000), incluye entre los objetivos del sistema "proteger paisagens naturais e pouco alteradas de notável beleza cênica” (Art. 4, inciso VI). La ley española (Ley 42/2007, de Patrimonio Natural y Biodiversidad), establece como uno de sus principios inspiradores La conservación y preservación de la variedad, singularidad y belleza de los ecosistemas naturales, de la diversidad geológica y del paisaje" (Art. 2, inciso d). Las negritas son añadidas por los autores de este trabajo.

3 La participación social en la labor de mantener un medio ambiente sano para el bien común está bastante explícita en las Constituciones. Por ejemplo, la de Brasil recoge en su Articulo 225 " Todos têm direito ao meio ambiente ecologicamente equilibrado, bem de uso comum do povo e essencial à sadia qualidade de vida, impondo-se ao Poder Público e à coletividade o dever de defendê-lo e preservá-lo para as presentes e futuras gerações". La Constitución de España recoge en su Artículo 45.2 "Los poderes públicos velarán por la utilización racional de todos los recursos naturales, con el fin de proteger y mejorar la calidad de la vida y defender y restaurar el medio ambiente, apoyándose en la indispensable solidaridad colectiva". Las negritas y subrayados son obra de los autores de este trabajo. 
visión de "burbuja" intocable de los primeros ENP para otra que toma en cuenta su inclusión como un elemento más del espacio geográfico que no puede permanecer al margen de sus procesos. También el hecho de que muchos de ellos estén habitados, por lo que se debe compaginar la conservación con el aprovechamiento de los recursos, ha sido decisivo en este viraje. Es en este contexto general donde se debe encuadrar el nacimiento y la aplicación de la noción de Custodia del Territorio, que en el ámbito de la conservación ha cobrado auge en los últimos años.

\section{Discutiendo el concepto de custodia del territorio}

Aunque sea en los últimos años cuando ha alcanzado mayor notoriedad y se ha extendido por más lugares, la denominada "Custodia del Territorio" existe desde hace bastante tiempo. El término deriva del inglés Land Stewardship y tiene su primer origen en los Estados Unidos, cuando en 1891 es fundada la entidad The Trustees of Reservation en el Estado de Massachussets (Basora y Sabaté, 2006). Muy conocido es el caso del National Trust en el Reino Unido, nacido en 1895 y que actualmente goza de gran prestigio social, con más de 3 millones de socios y colaboradores.

Intuitivamente, sin echar mano de ningún texto académico ni consultar glosarios, el concepto evoca el hecho de que “alguien” (particular o público, una persona o una entidad) “vigila” o “custodia” una porción de territorio, que por deducción debe ser suya o estar relacionado con ella de algún modo. No se conoce a priori la finalidad de la "custodia" aunque se intuye que consiste en protegerlo ante alguna amenaza o riesgo. Pues bien, si se consulta a organizaciones que trabajan con este asunto y a la literatura académica, se comprueba que las deducciones previas iban bien encaminadas. Por ejemplo, la Xarxa de Custodia del Territori de Catalunya (XCT), entidad fundada en 2003 y pionera en España en la misión de promover y desarrollar la Custodia del Territorio, la define como:

...conjunto de estrategias e instrumentos que pretenden implicar a los propietarios y usuarios del territorio en la conservación y el buen uso de los valores y los recursos naturales, culturales y paisajísticos. Para conseguirlo, promueve acuerdos y mecanismos de colaboración continua entre propietarios, entidades de custodia y otros agentes públicos y privados. (Basora y Sabaté, 2006).

Esta definición, por otra parte, es muy similar a la emanada de la Declaración de Montesquiu $^{4}$ en el año 2000, y que dice lo siguiente:

4 Este es el primer documento en España que hace “oficial” el concepto de Custodia del Territorio. 
Conjunto de estrategias diversas (de educación, de gestión, de mediación, de desarrollo comunitario...) que pretenden favorecer y hacer posible la responsabilidad en la conservación y uso adecuado (sostenible) del espacio terrestre, fluvial y marino y de sus recursos naturales, por parte de propietarios y usuarios de este territorio, y se dirige principalmente a la propiedad privada.

Por fin, autores como Donada et alt (2005; p.7) nos ofrecen una definición de Custodia del Territorio como "conjunto de estrategias y técnicas diversas que pretenden favorecer y hacer posible la responsabilidad de los propietarios o usuarios del territorio en la conservación de sus valores naturales, culturales y paisajísticos y en el uso adecuado (sostenible) de sus recursos".

Estas definiciones, tan similares entre sí, ratifican en buena parte la idea central antes expuesta. La Custodia del Territorio supone una estrategia de conservación del territorio y sus valores que normalmente implica el compromiso de particulares y de usuarios en esta tarea. La idea que late detrás de esto es bien simple: el poder público no tiene capacidad para proteger todos los valores naturales y culturales, valores que, por otra parte, no están solamente presentes en los ENP sino en todo el territorio en mayor o menor grado. Los terrenos particulares acogen valiosos elementos que sus propietarios y usuarios pueden proteger, contribuyendo a mantener un medio ambiente sano, necesario para el bien común de la sociedad. Es una forma de gestión complementaria de las oficiales regidas por el poder público, que facilita un mayor alcance territorial de la conservación y una mayor implicación social.

Por otra parte, la Custodia del Territorio denota que no sólo los ENP han de ser "protegidos". Todo el territorio ha de ser ordenado y tratado con el necesario cuidado a los procesos y ciclos naturales. El espacio cotidiano, donde la gente vive y trabaja, no puede ser de ninguna forma descuidado (Brown et alt, 2000). La existencia de ENP no supone una Patente de Corso para disponer libremente y sin cortapisa alguna de otros componentes del Espacio Geográfico.

Las definiciones también refuerzan otra idea previamente deducida. En la mayoría de los casos, un particular dedica voluntariamente un terreno de su propiedad a conservar sus elementos y dinámicas naturales, es decir, se compromete a "custodiar" para fines conservacionistas una parcela del territorio que es de su propiedad. Esto implica también a los usuarios del terreno (potencialmente todos nosotros), o lo que es lo mismo, a la sociedad en su conjunto.

En resumidas cuentas, es fundamental la idea de implicar a los agentes particulares en la conservación, pero no significa que actúen en solitario. De hecho, uno de los pilares de la Custodia del Territorio es la gestión coparticipada entre la propiedad y las llamadas Entidades de Custodia que son "organizaciones privadas sin ánimo de lucro o públicas de estructura diversa que participan activamente en la conservación del territorio, de sus recursos y de sus valores naturales, sociales o históricos" (Donada et alt, 2005; p12), y que por lo tanto tienen el papel de asesorar al propietario en la gestión, o bien directamente se encargan de ello. Usualmente, las entidades públicas participantes suelen ser de ámbito local (municipios sobre todo), 
mejores conocedoras del entorno. Y, desde luego, es una visión de la conservación bien alejada de aquella que propugnaba mantener intacto el espacio, bien al contrario:

La custodia del territorio es especialmente útil como un mecanismo de conservación en aquellos lugares donde el objetivo, más que la preservación absoluta y estricta de los valores naturales y/o culturales, es la gestión "meditada" de los recursos existentes. Así, se trata de un modelo perfectamente viable en zonas donde la tierra, las aguas y sus recursos se trabajen, exploten y comercialicen $\mathrm{y}$ de los cuales los propietarios o usuarios esperen un rendimiento” (Donada et alt, 2005; p7).

No es más que la puesta en práctica de los nuevos conceptos acerca de la gestión de los Espacios Naturales, donde ha de tenerse en cuenta la población local (residente en ellos o próxima a ellos) como un elemento imprescindible (Brown et alt hablan de modelos "inclusivos" de gestión). No significa esto que la visión anterior haya sido superada. En un lúcido trabajo, Reinaldo Arruda (1999) critica agudamente la cruel paradoja de proteger "de la gente", incluidos los residentes, aquellos Espacios Naturales que, si han sido declarados como tales, es precisamente por contar con sobresalientes valores naturales y culturales que han llegado hasta hoy justamente porque esa población residente ha moldeado esos lugares en conjunto con los factores ambientales (Paisaje, a fin de cuentas).

Las legislaciones ambientales elaboradas por los poderes públicos también recogen en sus textos la existencia de la Custodia del Territorio como opción de gestión del patrimonio natural. Es claro en el caso de España, que en su Ley de Patrimonio Natural y Biodiversidad aprobada en 2007 cuenta con un artículo completo sobre promoción del la Custodia del Territorio, el Artículo 72. Y por su parte, la Ley brasileña del Sistema de Unidades de Conservação aprobada en el año 2000, cuenta expresamente con una figura protectora denominada como "Reserva Particular do Patrimônio Natural" definida como "...área privada, gravada com perpetuidade, com o objetivo de conservar a diversidade biológica" (Art. 21). Esta figura es señalada como el ejemplo más próximo de Custodia del Territorio en Brasil (Basora y Sabaté, 2006; Oliveira, 2011), pues implica la acción voluntaria de un particular que desea proteger terrenos suyos y que para ello, establece una colaboración con entidades públicas y privadas (lo que no deja de ser un Acuerdo de Custodia, dicho sea de paso).

Teniendo en cuenta estas premisas, para el caso concreto de Brasil, parece claro que la figura de Reserva Particular do Patrimônio Natural es la más indicada para utilizar en aquellos lugares en que se pongan en funcionamiento iniciativas de Custodia del Territorio. Pero no necesariamente es la única opción. De hecho, el ejemplo a estudiar en este trabajo no se corresponde con esta figura protectora. El caso del Projeto Sempre Viva y del Parque Municipal de Mucugê (Figura 1) encaja a la perfección en el concepto de Custodia del Territorio. Proyecto nacido con la intención primera de poner a salvo una especie de Siempre-viva endémica de aquel lugar (Syngonanthus mucugensis giulietti), seriamente amenazada de extinción por la 
recolección con finalidad ornamental, ha derivado en la creación de un Parque Municipal $^{5}$ que no sólo preserva esta especie y toda la rica flora y fauna que posee, sino también busca mantener la Siempre-viva como fuente de ingresos para la población local a través de una exitoso trabajo de investigación para conseguir su cultivo in vitro en colaboración con universidades de la región. Además, ha puesto en marcha un trabajo intenso de educación ambiental para la población local y para los visitantes, y ha otorgado valor al legado cultural de la región, fuertemente vinculado al Garimpo (extracción artesanal de diamante). Por su origen en la escala local, por su afán en dar respuesta a un problema de conservación sin menoscabo del mantenimiento de las rentas de la población, por su exitosa colaboración con entidades académicas, por su valoración de la cultura propia, y principalmente por implicar directamente a la población local en su cuidado y gestión, es factible clasificar esta experiencia como ejemplo de Custodia del Territorio.

Para entender mejor el nacimiento de esta iniciativa y su razón de ser, el siguiente epígrafe ofrece un breve recorrido por la historia de Mucugê dentro del contexto general de la Chapada Diamantina, uno de los espacios protagonistas del "Ciclo del oro y las piedras preciosas" (Arruda, 1999) que han formado parte del devenir histórico de Brasil.

\section{Marco histórico: del Garimpo a la recolección de Siempre-Vivas}

El municipio de Mucugê está localizado en el Território de Identidade Chapada Diamantina, virtualmente en el centro geográfico del Estado de Bahia (ver Figura 1). Está compuesto por 24 municipios que ocupan juntos un área aproximada de 30.921 $\mathrm{Km}^{2}$, con una población de 376.467 habitantes, de los que el 50,23\% vive en la zona rural (www.territoriosdacidadania.gov.br).

La región de la Chapada Diamantina tiene una gran importancia estratégica en la gestión de recursos naturales del Estado. En primer lugar, en función de su elevada biodiversidad, con la presencia de especies raras de fauna y flora, pero también por sus peculiares características geológicas, geomorfológicas e hidrológicas (estratégico nudo de dispersión de aguas y fuente de los principales ríos que abastecen las ciudades baianas). Antigua área de explotación mineral (primero el oro, después los diamantes que le dieron nombre), la región ha vivido ciclos económicos

La Ley del Sistema de Unidades de conservación de Brasil recoge en su Artículo 8 la figura de Parque Nacional, si bien en su Artículo 11.4 indica que " As unidades dessa categoria, quando criadas pelo Estado ou Município, serão denominadas, respectivamente, Parque Estadual e Parque Natural Municipal". 
diferenciados ${ }^{6}$ (Seabra, 1991), cobrando fama hoy día por la agricultura empresarial tecnificada y el desarrollo del turismo.

Inicialmente habitada por los indios Maracás, a la región se le otorgaba poca importancia en los primeros años de la colonización. Era una barrera montañosa a atravesar o a flanquear en el proceso de desbravamento del Sertão. Si bien existían evidencias de la presencia de diamantes desde mediados del Siglo XVIII, su explotación fue prohibida por la Corona Portuguesa (Carta Regia de 1731) para evitar la competencia con los yacimientos de Minas Gerais, estratégicos por aquel entonces (Batista, 2007). No obstante, había enclaves ocupados ya entonces, ligados a yacimientos auríferos a partir de la primera década del Siglo XVIII, marcando el inicio de la llegada de los Bandeirantes y exploradores.

Figura 1. Localización del Área de Estudio.
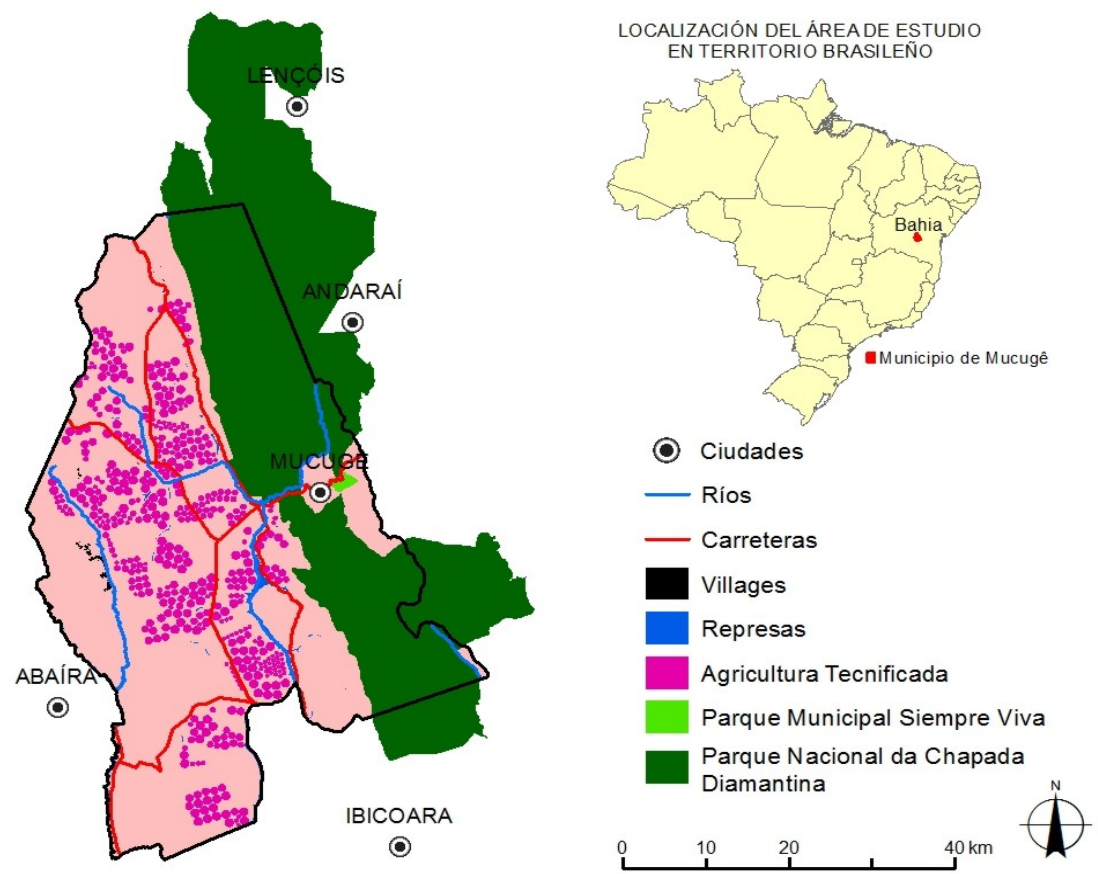

Fuente: Souza (2013)

6 Senna y Boratto (2006), en su esclarecedor estudio síntesis sobre el Parque Nacional de la Chapada Diamantina, hablan de "Chapada Aurífera", "Chapada Diamantífera" y "Chapada Turística", en alusión muy certera a los sucesivos ciclos de auge y decadencia de las que han sido testigos estas tierras. 
En la primera mitad del Siglo XIX, la colonización fue impulsada por el descubrimiento de diamantes en las inmediaciones del Rio Mucugê, lo que estimuló la ocupación de la región por parte de comerciantes, colonos, religiosos (principalmente Jesuitas) y extranjeros, que fundaron pequeños poblados, se diseminaron por las villas existentes y fundaron otras nuevas (www.bahia.com.br). Simbólicamente, el año 1844 figura como marco temporal que da inicio a la fiebre de los diamantes con el descubrimiento de valiosas gemas precisamente en el río Mucugê (Batista, 2007). Algunas de estas villas se transformaron después en municipios debido al crecimiento demográfico, entre ellos el propio Mucugê, Andaraí, Lençóis y Palmeiras, que acabaron constituyendo la región de las Lavras Diamantinas. El censo de 1872 informa de una población de 15.100 habitantes en Mucugê (Batista, 2007). Estimaciones apuntan que en pleno auge de la explotación de diamantes, esta población llegó a 25.000 personas. Eran los tiempos dorados del Garimpo, nombre que se dio a la técnica artesanal de búsqueda de diamantes en las gravas y arenas de los ríos y a la forma de vida de los buscadores de diamantes y su legado cultural, aún presente en el territorio.

Sin embargo, los tiempos de gloria y riqueza duraron bastante poco. Hacia 1870, el descubrimiento de nuevos yacimientos diamantíferos en África del Sur hizo disminuir el interés mundial por los diamantes baianos. La competencia de la producción sudafricana, aliada a los métodos primitivos de extracción aún por entonces utilizados en la Chapada Diamantina, hicieron caer la producción a índices mínimos. No hubo un abandono total gracias al carbonado o diamante negro, variedad rarísima usada para fines industriales, principalmente en la fabricación de brocas perforadoras, cuya demanda aumentó notablemente en la época por la construcción del Canal de Panamá, aliviando temporalmente la situación. A mediados de la década de 1920, la llegada del diamante sintético puso fin gradualmente a la producción de la gema, quedando apenas una pequeña producción artesanal en los aluviones.

De los años 1930 en adelante, con la caída de los precios del diamante, la baja productividad y el alto coste de producción, la Chapada Diamantina perdió lo poco que aún le quedaba de importancia política y económica, entrando así las Lavras Diamantinas en un largo período de retroceso económico y abandono que alcanzó su peor momento entre el final de los años 1960 y el inicio de los años 1970.

Con el éxodo de los moradores de los municipios de la Chapada hacia la región Sur y Sudeste de Brasil, principalmente hacia el Estado de São Paulo, se produjo una drástica reducción de la población residente y Mucugê llegó a tener menos de 400 habitantes (Batista, 2007). Esta población superviviente a la decadencia de la producción de diamantes se vio obligada a buscar una nueva alternativa de supervivencia. La hallaron en la recolección de Siempre-viva (Syngonanthus mucugensis giulietti), una especie vegetal endémica de la región de gran valor ornamental por poseer la rara virtud de permanecer lozanas aún después de muchos años de ser cortadas, de ahí su nombre (Figura 2).

La recolección de Siempre-vivas, principalmente destinadas a exportación para Japón, Estados Unidos y Europa, pasó a ser la principal fuente de recursos de la población residente y de los pueblos próximos, situación ésta que persistió durante 
más de treinta años (www.projetosempreviva.com.br). Esto fue posible en virtud del alto valor de estas bellas flores en el mercado internacional. En la década de 1960 la extracción anual era superior a 30 toneladas. Actualmente, este volumen no supera las cinco toneladas, que son exportadas ilegalmente por tratarse de una especie endémica y amenazada de extinción.

Debido a la extracción predatoria y a la ausencia de proyectos de recuperación de áreas o acciones de fiscalización, la especie fue seriamente diezmada en muchos campos hasta llegar a una situación de riesgo de extinción, agravada por los frecuentes incendios que asolan la región y dificultan aún más su preservación.

Figura 2. Siempre-vivas
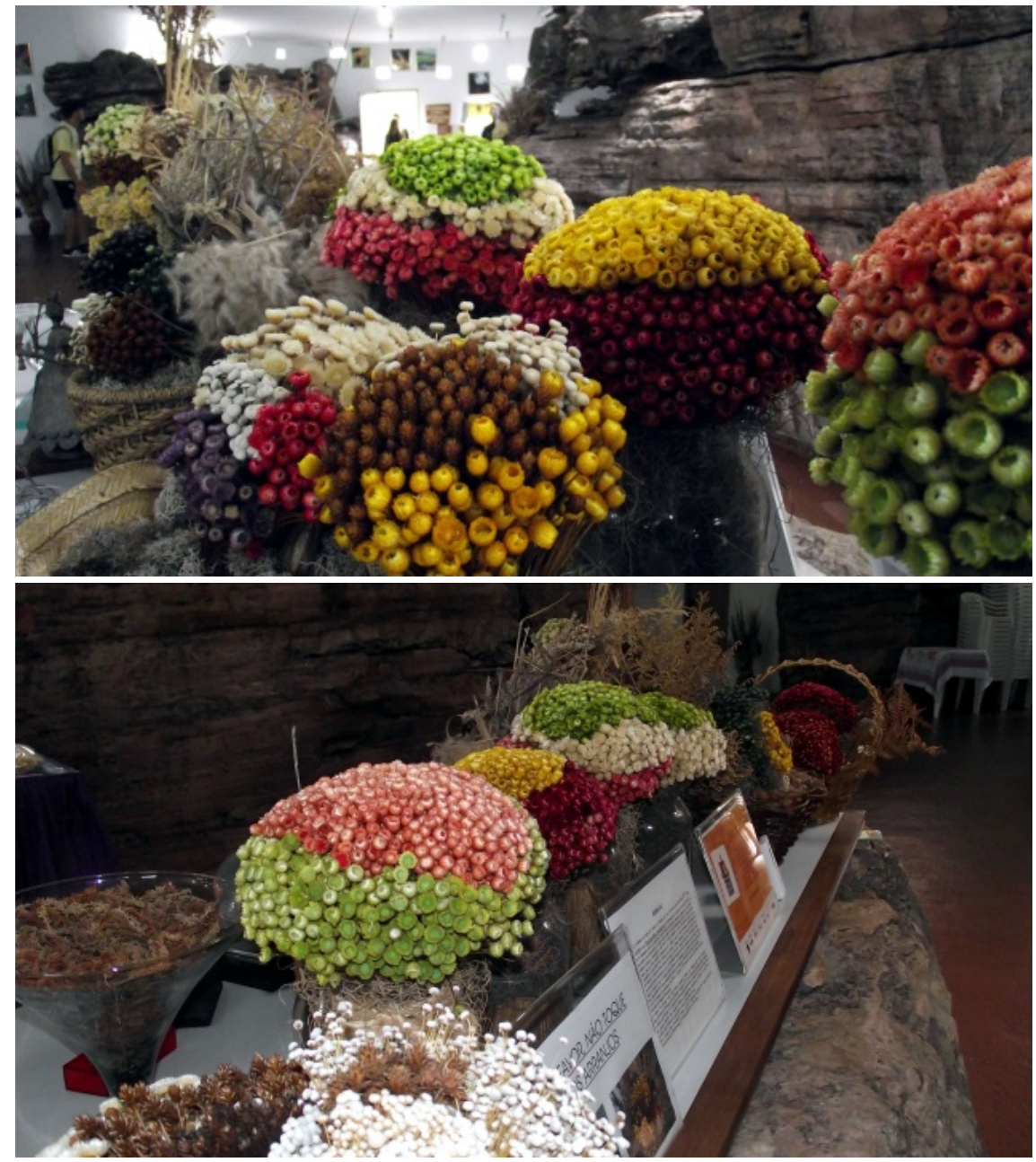

Fuente: Cabalar y Machado (2013) 
A caballo entre la década de 1980 y la de 1990, Mucugê comienza un cierto resurgir, que más tarde se consolidará, ligado a la agricultura irrigada y tecnificada. Primero a partir del cultivo de café, y posteriormente con una producción hortícola altamente capitalizada y mercantilizada basada en patata, cebolla y repollo, muy competitiva y lucrativa (buen ejemplo de Agribusiness). También ha ayudado el turismo. La declaración del Parque Nacional de la Chapada Diamantina en 1985 (del que el 52\% de su superficie pertenece al Término Municipal de Mucugê) y la inclusión del núcleo urbano de Mucugê en el catálogo de bienes del IPHAN (Instituto do Patrimônio Histórico e Artístico Nacional) desde 1980, colocaron la región en el mapa para muchos brasileños y extranjeros. La extraordinaria belleza paisajística, el elevado valor de la biodiversidad, y el legado histórico y cultural convierten estas tierras en apetecible destino, si bien aún muy mejorable en cuanto a infraestructuras y productos turísticos, y con algunos indeseables impactos sociales (véase, a tal efecto, el magnífico estudio de Brito, 2005). A día de hoy, la población total del municipio de Mucugê es de 10.545 habitantes, de acuerdo con los datos del Censo Demográfico de 2010 (IBGE, 2010).

\section{Nacimiento del Projeto Sempre Viva. Motivos, actores implicados, objetivos}

En honor a la verdad, apenas existe bibliografía específica sobre el proyecto y el Parque analizados en este trabajo. La fuente principal de información hay que buscarla en la propia página web del proyecto (lamentablemente ya desactivada), y especialmente en un Estudio de Caso elaborado por el IBAM (Instituto Brasileiro de Administração Municipal), elaborado por Marcia Batista el año 2007, que tuvo como objetivo analizar precisamente el Projeto Sempre Viva. Documento realmente muy completo que presenta con detalle los orígenes del proyecto, sus características, sus acciones y sus resultados, ha sido y será profusamente citado en este artículo.

El Projeto Sempre Viva nació por una parte con la intención de crear una alternativa para la generación de renta de la población más empobrecida, especialmente a través del artesanado producido de forma sostenible; y por otra parte con el objetivo de garantizar la supervivencia de la especie, repoblando los campos de donde prácticamente había desaparecido. Conviene recordar que tras la caída de la explotación del diamante, la región quedó sumida en una larga etapa de decadencia económica y demográfica, y para los pocos que aún quedaron allí, la recolección y venta de Siempre Vivas pasó a ser su única alternativa de sostén.

En 1993 la Prefeitura Municipal (Ayuntamiento) de Mucugê, por medio de una colaboración con la Pontificia Universidade Católica do Salvador (UCSal) inició un trabajo conjuntamente con la población local, apoyado por la Comisión de Medio Ambiente del municipio definiendo prioridades y acciones, entre ellas las de educación ambiental. Como resultado, la protección de la Siempre-viva y la creación de una unidad de conservación para abrigar el proyecto surgieron como metas principales a ser alcanzadas, proceso culminado seis años después. 
Lo más importante de todo, y que ha convertido este proyecto en rompedor, es la directa implicación de la población local en él. No deja de ser loable que precisamente hayan sido los ex-recolectores de Siempre Viva los principales gestores de la iniciativa ${ }^{7}$, y que ésta se haya permeado en la población de Mucugê hasta el extremo de hacer suyo el proyecto, una seña de identidad para el municipio visible en todo Brasil. Muchos ENP intentan conseguir la comunión con la población local sin éxito, y en Mucugê se ha conseguido. Es pues una iniciativa totalmente local: nace en Mucugê (Prefeitura: poder local), y se infiltra por toda la población mediante un concienzudo trabajo de educación ambiental. Las Universidades de la región colaboran con Know How científico a la hora de perseguir el doble objetivo conservación-desarrollo local (repoblación y cultivo con fines de conservación y lucrativos al mismo tiempo).

Como se puede apreciar en la Figura 1, el Parque Municipal ocupa una posición tal que le permite hacer la función de corredor ecológico, conectando dos extremidades del Parque Nacional de la Chapada Diamantina. Además está localizado en la principal área de recarga de los ríos de Contas y Paraguaçu, dos de los principales ríos del Estado de Bahia $^{8}$, y acoge especies nativas de la región que residen en ecosistemas peculiares asociados a locales de altitud, con cotas superiores a $1.200 \mathrm{~m}$ cuya vegetación predominante está asociada a campos de altitud (Campos Gerais) y dominios rupestres, establecido por Clements (1949, citado por el Gobierno del Estado da Bahia, 1993) como "Comunidad Reliquia", y por Veloso\&Goes-Filho como "Refugio Ecológico" (página web del proyecto). La biodiversidad y el grado de endemismos es tan alto que, incluso entre cumbres situadas a pocos kilómetros en línea recta, es frecuente hallar especies totalmente distintas y que sólo se encuentran allí (Senna et alt, 2006).

A todo esto cabe añadir el paisaje. El Parque Municipal de Mucugê constituye un bello escenario y un patrimonio de elevado valor para el municipio. Las cascadas (cachoeiras) de Tiburtino y Piabinha forman un conjunto atractivo entre los cañones formados en los lechos de los ríos, llamando la atención de los más de 14.000 visitantes que el parque recibe como media anualmente.

La creación del parque se produjo entre los años 1996 y 1999, fomentada por el Programa Nacional do Meio Ambiente (PNMA), resultando en el convenio MMA/PNMA/PED 96CV00027/96, que tuvo como partes el Ministerio de Medio Ambiente, el Gobierno del Estado da Bahia, la Prefeitura Municipal de Mucugê y la Pontificia Universidade Católica do Salvador.

Como objetivos generales fueron propuestos (Batista, 2007):

\footnotetext{
La tendencia de gran parte de los ENP a ignorar que la población local saca desde hace generaciones un provecho de los recursos naturales es un hecho frecuente. En este caso, la supervivencia dependía de la recolección de Siempre Vivas, sin una "mala intención" premeditada. Juiciosamente, el trabajo de educación ambiental realizado ha abierto los ojos de los recolectores y les ha hecho cambiar totalmente su punto de vista, sin conflictos inútiles. 
- Asegurar la supervivencia de la Siempre-viva nativa en la región, de gran valor comercial, promoviendo su cultivo para proporcionar alternativa económica a la población, prioritariamente las familias de ex-recolectores, así como la repoblación de los campos donde esta especie había sido diezmada;

- Preservar los recursos naturales e históricos (ciclo diamantífero) del municipio de Mucugê, a través de la creación de una unidad de manejo sostenible.

Estos objetivos fueron a su vez desdoblados en metas y estrategias específicas, conforme destacado en el convenio (Batista, 2007):

- Identificación y delimitación de áreas potenciales de ocurrencia de especies nativas de valor comercial, específicamente los campos de altitud donde se encuentran las Siempre-vivas;

- Desarrollo de tecnologías de cultivo y reproducción de Siempre-vivas para promover su comercialización sostenible y repoblar los campos donde la planta fue diezmada;

- Implantación de una Unidad de Cultivo Experimental con la creación de campos experimentales de producción de la Siempre-viva en su hábitat;

- Implementación de una Unidad de Conservación para proteger los campos de Siempre-viva y otros recursos naturales e históricos del municipio (el Parque Municipal);

- Implementación de un Banco de Datos y de un Sistema de Información Geográfica para el control de la producción y seguimiento de las áreas de ocurrencia de la especie;

- Promoción de programas de educación ambiental para modificar el cuadro generado por prácticas extractivas inadecuadas y para concienciar a la población sobre la importancia de la preservación y prepararla para el ejercicio de actividades sostenibles.

Los objetivos y metas resumen bien la razón de ser del proyecto y sus aspiraciones: el doble eje conservación-desarrollo local ligado a la recuperación de la Siempre viva para reintroducirla en su entorno natural y para servir de fuente de ingresos para la población local.

\section{El Projeto Sempre Viva hoy}

A diferencia de tantos otros proyectos de similares características e intenciones, el Projeto Sempre Viva se ha consolidado con el tiempo, al punto de ser a día de hoy una referencia y un ejemplo a seguir. El proyecto ha sabido aunar la protección, la educación ambiental y el desarrollo local, y además ha sido capaz de auto-financiarse, de manera que no depende de ayudas externas sino de sí mismo y de su propio desempeño. Es, de hecho, el único proyecto superviviente entre los que se presentaron en el año 1996 en la línea PED (Projetos de Execução Descentralizada) auspiciado 
por el Ministerio de Medio Ambiente de Brasil. Los autores de este trabajo han tenido oportunidad de conversar personalmente con el actual Secretario de Medio Ambiente del Municipio de Mucugê, que es además ex-recolector de Siempre-viva y una de las verdaderas Alma Máter del proyecto. Según sus palabras, el proyecto gira actualmente en torno a tres ejes principales. Son los siguientes:

a) Investigación para la conservación y el desarrollo:

El Projeto Sempre Viva, como ya se ha indicado, tiene su principal razón de ser en la conservación de la variedad endémica de Siempre-viva de Mucugê. A partir de aquí se ha construido una provechosa colaboración con las Universidades Federal de Bahia (UFBA) y Estadual de Feira de Santana (UEFS) que ha tenido una doble perspectiva. ${ }^{9}$ En primer lugar, estudiar sobre el terreno la Siempre-viva, en especial la localización de los enclaves donde ésta crece. Este trabajo fue realizado mediante posicionamiento GPS por varios ex-recolectores que conocían perfectamente donde crecen estas plantas y habían sido previamente entrenados en el uso de los dispositivos de localización. Este trabajo se extendió en etapas posteriores al conjunto de la flora presente en el área, extremadamente rica y variada, que destaca por la gran cantidad de endemismos casi única en el mundo (Senna et alt, 2006). Como resultado, se ha generado una vasta literatura científica sobre las características botánicas de este espacio. El segundo aspecto tiene relación tanto con la conservación como con el desarrollo local. La UEFS ha puesto en marcha un sistema de reproducción y cultivo in vitro de la Siempre-viva, con un éxito total. La intención es doble: preservar la especie intentando reintroducirla en su entorno natural, y conseguir una técnica de cultivo que permita comercializarla como planta ornamental para ser, de nuevo, fuente de renta para la población local. La venta de Siempre-viva ha sido durante mucho tiempo el sustento de mucha gente en Mucugê tras el final definitivo del ciclo del diamante, y con este plan se intenta que siga siendo fuente de riqueza sin poner en riesgo la existencia de la propia especie. En palabras del secretario de Medio Ambiente, sólo falta un último paso en este feliz proceso: que la técnica de cultivo sea traspasada a los ex-recolectores, proceso que al parecer no tardará mucho en completarse.

b) La educación ambiental y la valoración del patrimonio natural y cultural:

El intenso y exitoso desempeño en el ámbito educativo del Projeto Sempre Viva ha logrado trascender a todo Brasil. El primer y necesario paso fue la labor educativa con el propio habitante de Mucugê, y más aún, con los ex-recolectores de Siempre Viva. Estas personas veían seriamente amenazado su porvenir con la prohibición de recolectar y pasaron después a ser los gestores del proyecto, a sentirlo como parte de si, y a transmitir sus conocimientos sobre la naturaleza y la cultura del lugar a

9 Inicialmente, el convenio de colaboración para la conservación de la Siempre-viva había sido realizado con la Pontificia Universidade Católica do Salvador (UCSal), pero al cabo de un tiempo se apartó del proyecto. 
multitud de centros de todos los niveles educativos que acuden a Mucugê desde todo el Estado de Bahia y desde varios puntos de Brasil. Ese trabajo de toma de conciencia ambiental ha cristalizado, al mismo tiempo, en el hecho de que Mucugê sea a día de hoy uno de los municipios más limpios y que haga selección de residuos, instalándose allí la primera planta de tratamiento y compostaje del Estado de Bahia (Ribeiro et alt, 2012). El Parque Municipal ofrece, además, visitas guiadas para estudiantes e investigadores, y un espacio propio para alojamiento de los mismos. El parque completa sus instalaciones educativas con una bien elaborada exposición interpretativa de los principales aspectos naturales y culturales de la región. Pero la educación ambiental no ha repercutido solamente en una conducta diferente hacia la naturaleza, también ha puesto en valor el patrimonio natural y cultural. Los habitantes de Mucugê se encuentran hoy legítimamente orgullosos de su municipio y de su cultura, pues el proyecto se ha encargado de transmitir y hacer partícipes a todos sus habitantes de su rica herencia histórica de la etapa diamantífera, que se plasma tanto en el bello conjunto urbano de Mucugê (declarado como Monumento Histórico Nacional en 1980) como en el legado cultural del Garimpo (extracción artesanal de diamantes a pequeña escala), disperso por todo el territorio y que forma parte de los atractivos turístico-educativos auspiciados por el proyecto. Precisamente el proyecto ha puesto en funcionamiento el llamada Museu Vivo do Garimpo, una instalación exclusivamente centrada en la puesta en valor de la antigua actividad extractiva del diamante y de su forma de vida mediante un museo y exposición interpretativa, que incluye un repaso por la historia de la explotación del diamante y su importancia en la economía mundial. Se completa con una choza tradicional de Garimpeiro, muy próxima al museo, donde se exhiben todos sus instrumentos de trabajo y los útiles domésticos.

c) Los Sistemas de Información Geográfica como herramientas de gestión:

Con anterioridad se ha mencionado que uno de los pilares del proyecto es la localización de las áreas donde crece la Siempre-viva, y que este trabajo era realizado con GPS. Pues bien, derivado de ello se ha puesto en marcha toda una base de datos espacial vinculada a un Sistema de Información Geográfica (GIS) que se ha constituido en herramienta de primer orden para la gestión ambiental no sólo del Parque Municipal sino también de la totalidad del municipio de Mucugê. En efecto, la gestión del municipio utiliza la herramienta GIS derivada del proyecto con notables resultados prácticos, y de hecho está ayudando a los empresarios del Agropolo (gran área de agricultura tecnificada que es hoy día el otro puntal económico) a monitorear mejor sus cosechas y demarcar con precisión los terrenos, incluyendo aquellas áreas reservadas para preservación (Áreas de Preservación Permanente) que contempla la legislación brasileña. Pero Mucugê también se vale de esta valiosa herramienta de trabajo para fiscalizar y monitorear la gestión municipal en su conjunto, tanto la urbana como la rural. 


\section{Conclusiones}

El Projeto Sempre Viva se ha convertido en una de las experiencias más exitosas de conservación de la naturaleza a escala local de todo Brasil. El proyecto ha conseguido éxito en su objetivo primordial, que es la conservación de la Siempre Viva, mediante una colaboración con universidades e investigadores que ha sido ejemplar. Pero además ha sido capaz de compaginar la conservación con el desarrollo local al enfocar el proyecto a conseguir que la Siempre Viva continúe siendo una fuente de renta, no se ha quedado en la mera conservación (con ser ésta sumamente importante), y además ha puesto en valor el lugar y sus valores naturales y culturales hasta hacerlo conocido y respetado en todo Brasil. Pero el gran secreto del proyecto, como ha confesado el secretario de Medio Ambiente de Mucugê, es un hecho largamente anhelado por todos los Espacios Naturales y pocas veces alcanzado: ha conseguido implicar totalmente a la población local, una población que lo ha impulsado desde el principio y lo ha incorporado como algo propio, como algo suyo, como una seña de identidad que les hace sentirse orgullosos de ser habitantes de Mucugê. En sus propias palabras, la gente se ha "puesto la camiseta" del proyecto y ha recuperado la autoestima y el orgullo de ser y pertenecer a Mucugê.

\section{Referencias bibliográficas}

Arruda, R. (1999): "Populações tradicionais" e a proteção dos recursos naturais em Unidades de Conservação. Ambiente \& Sociedade, Ano II, N5. pp 79-92.

Basora Roca, X.; Sabaté i Rotés, X. (2006): Custodia del Territorio en la práctica. Manual de introducción a una nueva estrategia participativa de conservación de la naturaleza y el paisaje. Xarxa de Custodia del Territori y Fundació Territori i Paisatge. 80 pp.

Batista, M. A. (2007): Estudo de caso: projeto sempre viva: Mucugê-Bahia/Márcia Aguiar Nogueira Batista; supervisão de Marlene Fernandes; coordenação de Carlos Alberto Silva Arruda. - Rio de Janeiro: IBAM. 61 pp.

Brown, J.; Mitchell, B. (2000): The Stewardship approach and its relevance for Protected Landscapes. The George Wright Forum, Vol. 17 Nº1. pp 70-79.

Corraliza RodrigueZ, J. A.; Garcia Navarro, J.; Valero Gutiérrez del Olmo, E. (2002). Los Parques Naturales en España: conservación y disfrute. Fundación Alfonso Martín Escudero, Madrid.

Diego Liaño, C.; García Codrón, J. C. (2007). Los Espacios Naturales Protegidos. DaVinci, Mataró,

Donada, L.; Ormazábal, M. (2005). Custodia del Territorio: un modelo de gestión dentro de La Red Natura 2000. Proyecto fin de Máster, Universidad Autónoma de Madrid, Universidad Complutense de Madrid y Universidad de Alcalá.

Fernandes Neto, Otoniel, Theodoro Sampaio e a Chapada Diamantina, Edição do Autor, Brasília, DF, 2005

IBGE - Instituto Brasileiro de Geografia e Estatística. Censo Demográfico 2010. 
Mulero Mendigorri, Alfonso. La protección de Espacios Naturales en España: antecedentes, contrastes territoriales, conflictos y perspectivas. Editorial Mundi-Prensa, Madrid, 2002.

Oliveira do Prado, R. C. (2011): Las Reservas Particulares do Patrimonio Natural (RPPN) de Brasil y la Xarxa de Custòdia del Territori (XCT) de Catalunya como herramientas comunes para el Desarrollo Sostenible. Veredas do Direito, Vol. 8 N¹5. pp 165-190.

Página web del Projeto Sempre Viva: www.projetosempreviva.com.br

Portal Oficial de Turismo do Estado da Bahia. Disponible en: <http://www.bahia.com.br>.

Portal da Cidadania - Governo Federal do Brasil. Disponible en: $<$ http://www.territoriosdacidadania.gov.br>.

Ribeiro Tanan, Karla Christiane; CHAVES, Joselisa Maria. A educação ambiental como prática propositiva na intervenção das queimadas no município de Mucugê, Chapada Diamantina-Ba. I Seminario Nacional de Geoecologia e Planejamento Territorial e IV Seminário do Geoplan. Universidade Federal de Sergipe, 2012.

Seabra, Giovanni de F. Estudo Geomorfológico da Região Cárstica de Andaraí: uma contribuição à conservação. Dissertação de Mestrado em Geografia Física (mimeo). DGEO / CFCH / UFPE: Recife, 1991.

Senna Ganem, R.; Boratto Viana, M. (2006): História ambiental do Parque Nacional da Chapada Diamantina/Ba. Câmara dos Deputados, Brasília. 34 pp. 